\title{
Evaluating the effect of conditioned medium from endometrial stem cells on endometriosis-derived endometrial stem cells
}

\author{
Seyedeh Saeideh Sahraei ${ }^{1,2}$, Ali Kowsari ${ }^{1}$, Faezeh Davoodi Asl ${ }^{1}$, Mohsen Sheykhhasan ${ }^{1}$, Leila Naserpoor ${ }^{2}$, \\ Azar Sheikholeslami ${ }^{1}$ \\ ${ }^{1}$ Department of Mesenchymal Stem Cells, The Academic Centre for Education, Culture and Research, Qom, ${ }^{2}$ Department of Reproductive Biology, The \\ Academic Centre for Education, Culture and Research, Qom, Iran
}

\begin{abstract}
Endometriosis is a common, benign gynecological disease which is determined as an overspreading of endometrial tissue in exterior region of the uterine cavity. Evidence suggests that retrograde menstrual blood which contains mesenchymal stem cells with differential gene expression compared to healthy women may play a role in endometriosis creation. We aimed to identify whether the conditioned medium (CM) from menstrual blood-derived mesenchymal stem cells (MenSCs) of healthy women can affect the expression level of inflammatory and stemness genes of MenSCs from endometriosis women. Endometriosis-derived MenSCs (E-MenSCs) were treated with CM derived from healthy women's MenSCs (non-endometriosis derived MenSCs [NE-MenSCs]). Some CD markers were analyzed by flow cytometer before and after treatment compared with NE-MenSCs, and the expression level of inflammatory and stemness genes was evaluated by real-time PCR. E-MenSCs show different morphology in vitro culture in comparison with NE-MenSCs, which were changed in the presence of $\mathrm{CM}$, into a morphology more similar to normal cells and showed significant decrease expression of CD10 after CM treatment. In our results, the interleukin-1, cyclooxygenase-2, and hypoxia-inducible factor $1 a$ as inflamaturay genes and octamer-binding transcription factor 4, NANOG, and sex determining region Y-box 2 as stemness genes showed significantly different expression level in E-MenSCs after treating with CM. Our study indicates that the expression level of some inflammatory- and stemness-related genes which have differential expression in E-MenSCs compared with NEMenSCs, could be changed to normal status by using CM derived from NE-MenSCs.
\end{abstract}

Key words: Endometriosis, Mesenchymal stem cells, Conditioned Medium

Received August 23, 2021; 1st Revised September 9, 2021; 2nd Revised September 30, 2021; Accepted September 30, 2021

\section{Introduction}

Endometriosis is a benign gynecological disease that affects approximately $5 \%$ to $10 \%$ of women worldwide during

\section{Corresponding author:}

Azar Sheikholeslami (iD

Department of Mesenchymal Stem Cells, The Academic Centre for Education, Culture and Research, Oom 3713189934, Iran

E-mail: azareslami1o@gmail.com the years of reproduction $[1,2]$, and is described as the presence of endometrial tissues outside the uterine cavity, which leads to many complications such as chronic pelvic pain and infertility [3]. Since endometriosis is a complex disease with a multifactorial etiology, generally accepted theories reported for the causes of endometriosis include retrograde menstruation, vascular dissemination, metaplasias in pelvic cavity, and immune dysfunction. For instance, many shreds of evidence offer that immune responses are activated in endometriosis that lead to cellular changes in menstrual bloodderived mesenchymal stem cells (MenSCs) [3, 4]. Regarding 
the differences observed in MenSCs of endometriosis women in comparison with healthy women, it seems very important to evaluate these cells in different aspects including surface markers and gene expression levels $[2,4]$.

Some genes are differentially expressed in MenSCs from endometriosis patients which can be the leading reasons for the disease complications. These gene expression differences can help to diagnosis and treatment of endometriosis [5]. Inflammatory genes are mostly overexpressed in these patients which gradually leads to chronic inflammation and immune responses in lesion sites and the whole pelvic cavity [6]. Besides, the stemness genes, also known as pluripotency genes, are mostly expressed differentially in MenSCs from endometriosis women which can result in angiogenesis, migration, and invasion $[7,8]$. Since there is no proper and definitive treatment for this disease, it could be important to know the basic principles that cause it, such as differentially expressed genes, to find suitable treatment methods.

One of the main inflammatory actors is interleukin (IL)-1 $\alpha$. It is thought that IL- $1 \alpha$ is a primary inflammatory factor in immune situations that stimulates prostaglandin synthesis and induces $B$ cells to produce immunoglobulins. So, IL- $1 \alpha$ can play a significant role in the pathogenesis of endometriosis [9]. Also, cyclooxygenase-2 (COX-2) is an inducible enzyme that is produced by inflammatory mediators. It has been proved that expression of COX2 enhances in women with endometriosis. It has been proved that expression of COX-2 enhances in women with endometriosis. Consequently, the high levels of COX2 and Prostaglandins expression might be effective in endometriosis development [10]. Furthermore, aberrant expression of tumor necrosis factor (TNF)- $\alpha$ is identified to promote the cell proliferation and adhesion in endometriosis, because high concentrations of TNF- $\alpha$ induce the peritoneal macrophages to secrete inflammatory factors [11]. The inflammatory mediators such as IL-1 $\beta$ and TNF- $\alpha$ have key function in activating nuclear factor $\kappa \mathrm{B}(\mathrm{NF}-\kappa \mathrm{B})$ and hypoxia-inducible factor $(\mathrm{HIF}-1 \alpha)$ signaling pathway. These factors regulate immune response and high expression of $\mathrm{NF}-\kappa \mathrm{B}$ and HIF- $1 \alpha$ are observed in endometriosis [12].

On the other hand, stemness genes play an emerging role in endometrium regeneration in each woman ovarian cycle. In addition, pluripotency genes promote cell migration in endometriosis. It was proved that the expression of stemnessrelated factors such as the Spalt-like protein 4 (SALL4), NANOG, octamer-binding transcription factor 4 (OCT4), and sex determining region Y-box 2 (SOX2) are different in endometrial stem cells between endometriosis patients and healthy women $[13,14]$. OCT4 is an important factor for the survival of cell pluripotency and is identified to be highly expressed in endometriosis women [15]. In endometriosis, expression of the pluripotent transcription factor OCT4 encourages cell migration [7]. NANOG expression in undifferentiated stem cells and it is a critical regulator in cells pluripotency and differentiation by interaction with other factors $[16,17]$. Many studies revealed that SALL4 expression changes in numerous cancers and endometriosis $[18,19]$.

Since there is no proper and definitive treatment for endometriosis, it can be very important to know its etiology to find suitable treatment methods. Extensive investigations in recent years on mesenchymal stem cells-derived conditioned medium (MSCs-CM) lead our mind to the dynamic role of endometrial stem/progenitor cells and their derivatives in the treatment of endometriosis. Growth factors, extracellular vesicles, and bioactive agents in the secretion released by stem cells into the in vitro culture medium, known as the conditioned medium, have been shown to induce many cell processes and changes in gene expression, and eventually, tissue repair and regeneration through paracrine mechanisms [20-22].

Regarding the various functions reported for MSCs-CM, it can be expected that the conditioned medium from MenSCs of healthy women can affect the surface markers and gene expression of MenSCs from endometriosis women. So, the aim of this study was to evaluate the expression of inflammatory and stemness genes in endometriosis patients in the presence of CM from endometrial stem cells of healthy women.

\section{Materials and Methods}

\section{Patient selection}

This interventional experimental study was approved by Azad University ethics committee (IR.IAU.QOM. REC.1399.065) and written informed consents were obtained from individuals before enrolling in the study. The patient group comprised endometriosis women $(n=3)$ (stages III-IV) undergoing laparoscopy for infertility or pain and the control group consisted of healthy women (non-endometriosis subjects) ( $n=3)$. The inclusion criteria were as follows: (1) age ranging from 25 to 35 years in both groups; (2) history of ovulatory cycles with unregularly menstrual periods in endometriosis cases; (3) body mass index of $18-28 \mathrm{~kg} / \mathrm{m}^{2}$; (4) 
no hormonal treatments for at least the last 3 months; (5) no previous surgery for endometriosis women: (6) no history of malignancies or autoimmune diseases; and (7) evidence for deep endometriosis suggested by transvaginal ultrasound and magnetic resonance imaging (Fig. 1).

\section{MenSCs isolation and culture}

After selecting 3 proper cases in each groups, at least $2 \mathrm{ml}$ menstrual blood was collected by pipelle catheter during the second or third day of menstruation and were immediately transferred to the laboratory. Four folds of blood sample was added EDTA $0.5 \mathrm{mM}$, an equal volume of blood sample was added to Ficoll-Paque media (Lymphodex; inno-train, Kronberg, Germany) carefully and centrifuged at $600 \mathrm{~g}$ for 30 minutes at room temperature. Following density gradient centrifugation, plasma and platelets in the upper layer were removed and mononuclear cell layer remained undisturbed at the interface. The mononuclear cell layer was transferred to a sterile centrifuge tube and washed twice with phosphate buffered saline. Cell pellets were grown in Dulbecco's modified Eagle's low glucose (DMEM-LG) medium supplemented with $10 \%$ fetal bovine serum (Gibco, Grand Island, NY, USA) and $1 \%$ penicillin/streptomycin (Gibco) and incubated in $97 \%$ humidity, and $5 \% \mathrm{CO}_{2}$ at $37^{\circ} \mathrm{C}$. The isolated MenSCs from endometriosis and healthy women are defined as endometriosis-derived MenSCs (E-MenSCs) and nonendometriosis derived MenSCs (NE-MenSCs), respectively.

\section{Flow cytometry for mesenchymal stem cell markers}

To confirm the isolated cells as MenSCs, the expression level of positive cell surface markers (CD29, CD90, CD105, CD44, CD73, and CD10) and negative markers (CD34, CD45, CD133, CD38) for MenSCs were determined. FITCconjugated monoclonal antibodies against CD34, CD38, CD90, CD10, CD44, and CD133, as well as PE-conjugated monoclonal antibodies for CD105, CD45, CD29 and CD73 were purchased from BD Biosciences (San Jose, CA, USA). PE-conjugated anti-CD105 and were from R\&D Systems (Minneapolis, MN, USA). The entire corresponding isotypematched control antibodies were obtained from the same companies as their test antibodies. All the antibodies were used in flow cytometry experiments at the concentrations recommended by the manufacturers. Flow cytometry was performed using a FC500 flow cytometer (Beckman Coulter, Fullerton, CA, USA) and analyzed using Beckman Coulter CXP software.

\section{Preparation of conditioned medium and treatment}

For preparation of conditioned medium, NE-MenSCs at passages 3-4 were seeded in T-75 flasks at a density of $1 \times 10^{6}$ cells. Once the $70 \%-80 \%$ confluency was obtained, the culture medium was collected after 3 days of culture. To remove cells and cell debris, culture medium was centrifuged and filter-sterilized through a $0.22-\mathrm{m}$ filter, and then it was termed as a conditioned medium of MenSCs (MenSCs-CM). The aliquoted MenSCs-CM was stored at $-80^{\circ} \mathrm{C}$ till use. All in vitro experiments were performed using passages $3-5$ of MenSCs-CM.

Then, E-MenSCs were treated in three experimental groups with NE-MenSCs derived conditioned medium for 2, 4, and 6 days. Before and after CM treatment the cell lines was measured by flow cytometry assay and real-time PCR.

\section{Real-time polymerase chain reaction and gene expression analysis}

Total RNA was isolated from treated cells using "Gene All Kit (Gene All Biotechnology, Seoul, Korea) according to the manufacturer's instructions. RNA purity and quantity were assessed using Nanodrop 2000 spectrophotometer (Thermo

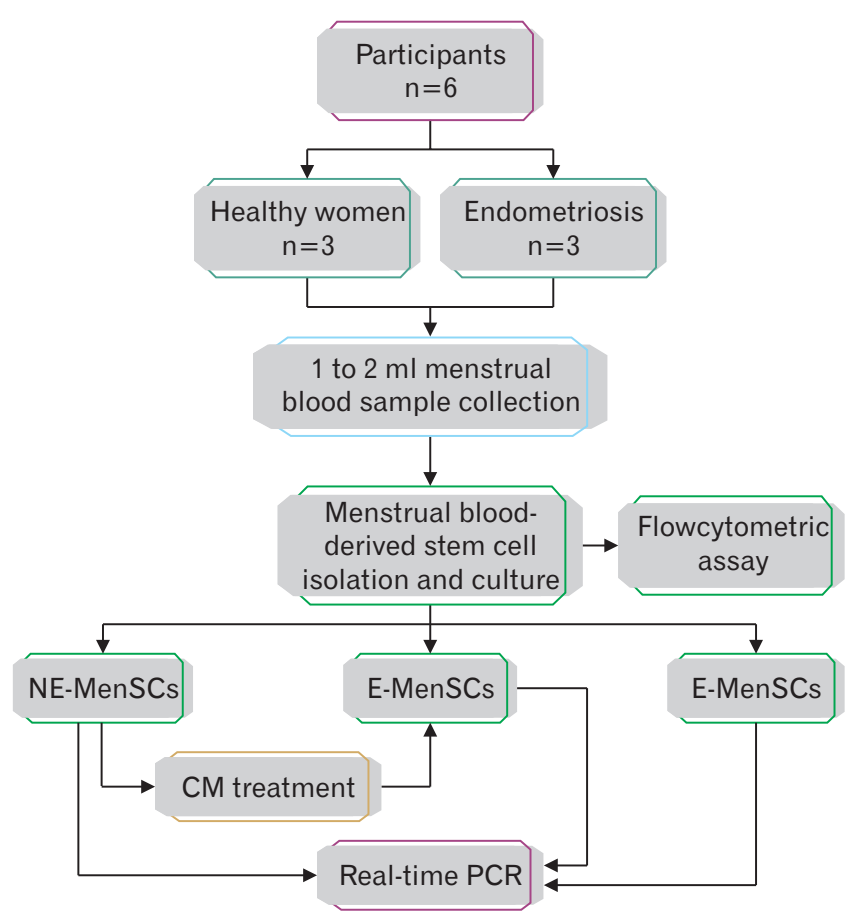

Fig. 1. The flowchart of the study design. NE-MenSCs, non-endometriosis derived menstrual blood-derived mesenchymal stem cells; E-MenSCs, endometriosis derived menstrual blood-derived mesenchymal stem cells; CM, conditioned medium. 
Fisher Scientific, Wilmington, NC, USA) at 260/280 nm. The reverse transcription was used to synthesize the firststrand cDNA using transcription Kit (Yekta tajhiz, Tehran, Iran)." Quantitative real-time PCR assays were performed in triplicate to evaluate the expression of selected genes (Table 1). For normalizing gene expression levels, glyceraldehyde3-phosphate dehydrogenase $(G A P D H)$ gene was used as an internal reference. The $2^{-\Delta \Delta C t}$ method was used to calculate the fold change of mRNA expressions for target genes. Realtime PCR was carried out using RealQ Plus Master Mix Green (Ampliqon, Odense M, Denmark) following the manufacturer's instructions. Briefly, a mixture comprised of $10 \mu \mathrm{l}$ SYBR green mix, $1 \mu \mathrm{l}$ of cDNA (250 ng), $1 \mu \mathrm{l}$ PCR forward primers and $1 \mu \mathrm{l}$ PCR reverse primer in $5 \mathrm{pmol} \mu \mathrm{l}$ 1 , and millipore water to achieve a final volume of $20 \mu \mathrm{l}$ was made. The sequences of primers are presented in Table 1 . The Threshold Cycle (CT) was determined manually for each run. Relative mRNA level was expressed as the relative fold change and calculated using the formula $2^{-\Delta \Delta \mathrm{CT}}=2-\left(\Delta^{\mathrm{CT}(\text { Sample })}-\right.$

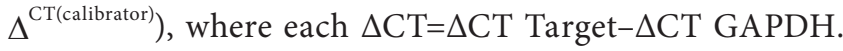
One sample without any treatment from the control group was designated as a calibrator. The quantification of mRNA was performed as a value relative to an internal reference for GAPDH.

Table 1. Primer pairs used in real-time PCR

\begin{tabular}{|c|c|c|c|}
\hline Gene & Sequence & Accession number & Product size (bp) \\
\hline \multirow[t]{2}{*}{$I L-1 \beta$} & F: TCTTCTTCGACACATGGGATA & NM_000576.3 & 183 \\
\hline & R: GTACAAAGGACATGGAGAACA & & \\
\hline \multirow[t]{2}{*}{$C O X-2$} & F: TCAGCCATACAGCAAATCCT & NM_000963.4 & 205 \\
\hline & R: TTGAAGTGGGTAAGTATGTAGTG & & \\
\hline \multirow[t]{2}{*}{$T N F-\alpha$} & F: GTCTGGGCAGGTCTACTTTGG & MH180383.1 & 172 \\
\hline & R: GTTCTAAGCTTGGGTTCCGAC & & \\
\hline \multirow[t]{2}{*}{$N F-\kappa B$} & F: GAAGTGCAGAGGAAACGTCAG & NM_001382627.1 & 147 \\
\hline & R: GAAGCTATACCCTGGACCTGT & & \\
\hline \multirow[t]{2}{*}{$H I F-1 \alpha$} & F: GGCGAAGTAAAGAATCTGAAG & NM_181054.3 & 209 \\
\hline & R: ACCATCCAAGGCTTTCAAATA & & \\
\hline \multirow[t]{2}{*}{ OCT4 } & F: GTTCTTCATTCACTAAGGAAGG & NM_001285986.2 & 101 \\
\hline & R: CAAGAGCATCATTGAACTTCAC & & \\
\hline \multirow[t]{2}{*}{$N A N O G$} & F: ACCTGAAGACGTGTGAAGATG & NM_001355281.2 & 187 \\
\hline & R: ATTAGGCTCCAACCATACTCC & & \\
\hline \multirow[t]{2}{*}{ SOX 2} & F: GGGAAATGGAAGGGGTGCAAAAGAGG & NM_003106.4 & 151 \\
\hline & R:TTGCGTGAGTGTGGATGGGATTGGTG & & \\
\hline \multirow[t]{2}{*}{ SALL4 } & F: GGGCAGCCACATGTCTCAGCA & NM_001318031.2 & 204 \\
\hline & R: GACATGACGTTCGGGAGCACC & & \\
\hline
\end{tabular}

IL-1 $\alpha$, interleukin $1 \alpha$; COX-2, cyclooxygenase-2; TNF- $\alpha$, tumor necrosis factor- $\alpha$; NF- $\kappa$, nuclear factor $\kappa$ B; HIF-1 $\alpha$, hypoxia-inducible factor $1 \alpha$; OCT4, octamer-binding transcription factor 4; SOX2, sex determining region Y-box 2; SALL4, Spalt-like protein 4.
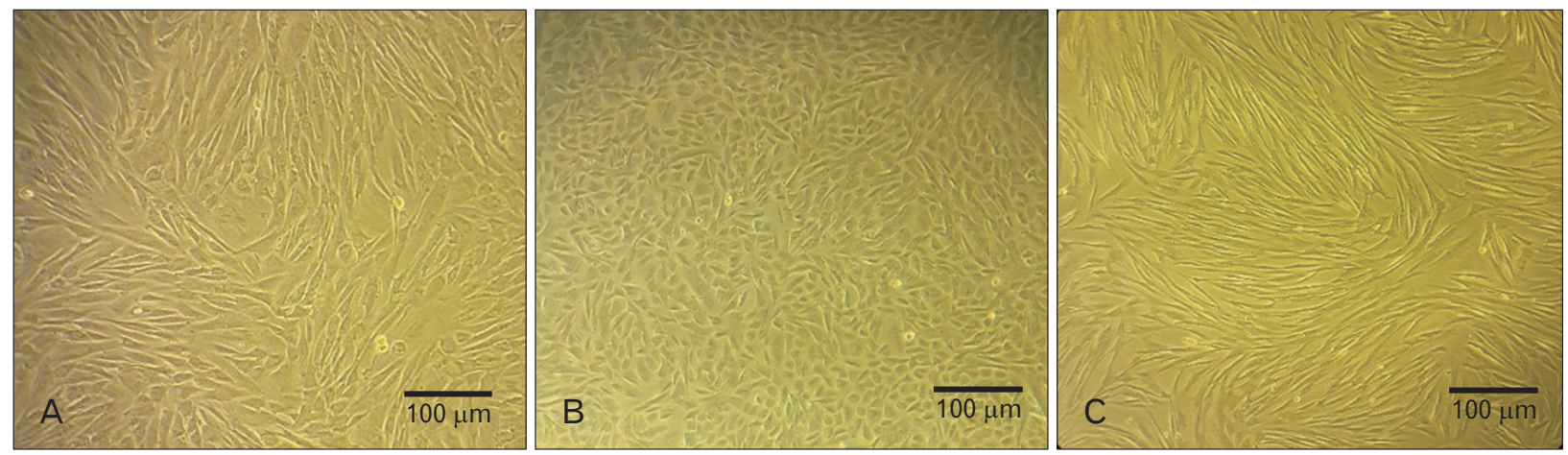

Fig. 2. Morphological difference between non-endometriosis derived menstrual blood-derived mesenchymal stem cells (NE-MenSCs) and endometriosis derived menstrual blood-derived mesenchymal stem cells (E-MenSCs). NE-MenSCs in 2D culture showed a fibroblast-like spindle-shaped morphology (A), while E-MenSCs were less stretched and more circular (B). After conditioned medium (CM) treatment, the cells changed to spindle-shaped morphology (C). 


\section{Statistical analysis}

Experimental data are expressed as the mean \pm standard error (SE) and compared by ANOVA. ANOVA using a multiple comparisons test was used to determine statistical significance, followed by the Tukey test. Statistical significance was set at $P \leq 0.05$. All experiments were performed in triplicate for technical and biological repetitions.
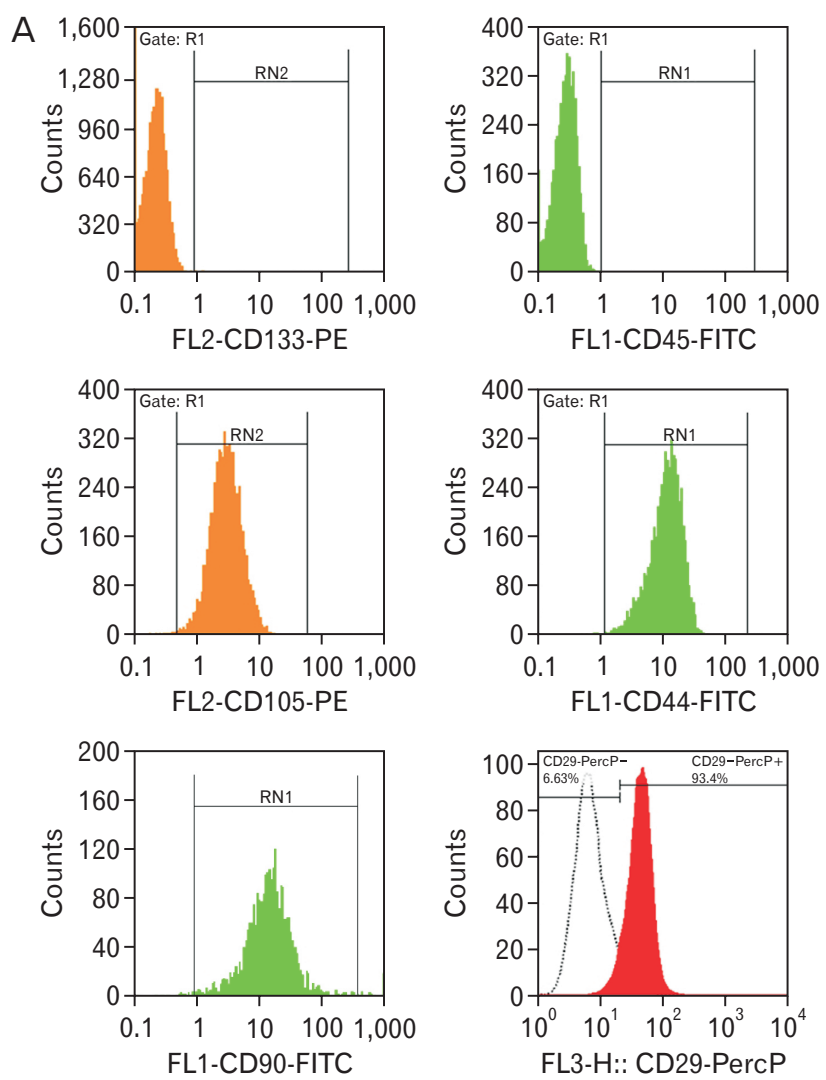
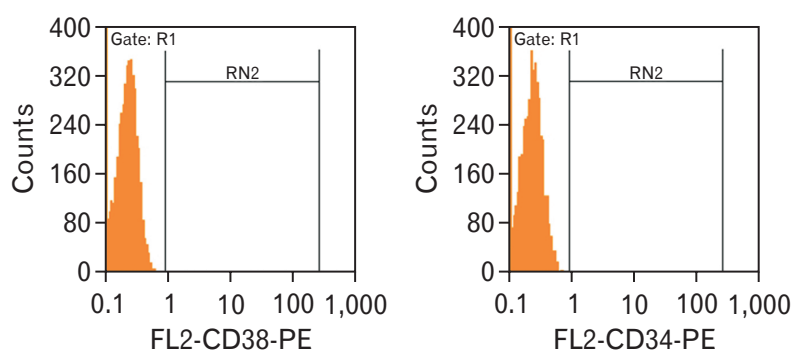

\section{Results}

\section{Morphology of MenSCs}

The MenSCs are morphologically similar to bone marrow-derived mesenchymal stem cells. In our study, microscopic observations showed that MenSCs from healthy women (NE-MenSCs) had fibroblast-like spindle-shaped morphology, while, E-MenSCs were less stretched and elongated (Fig. 2A, B). The morphology of E-MenSCs was
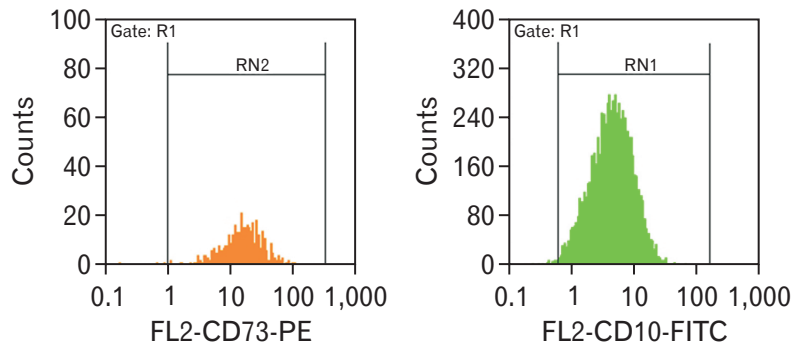

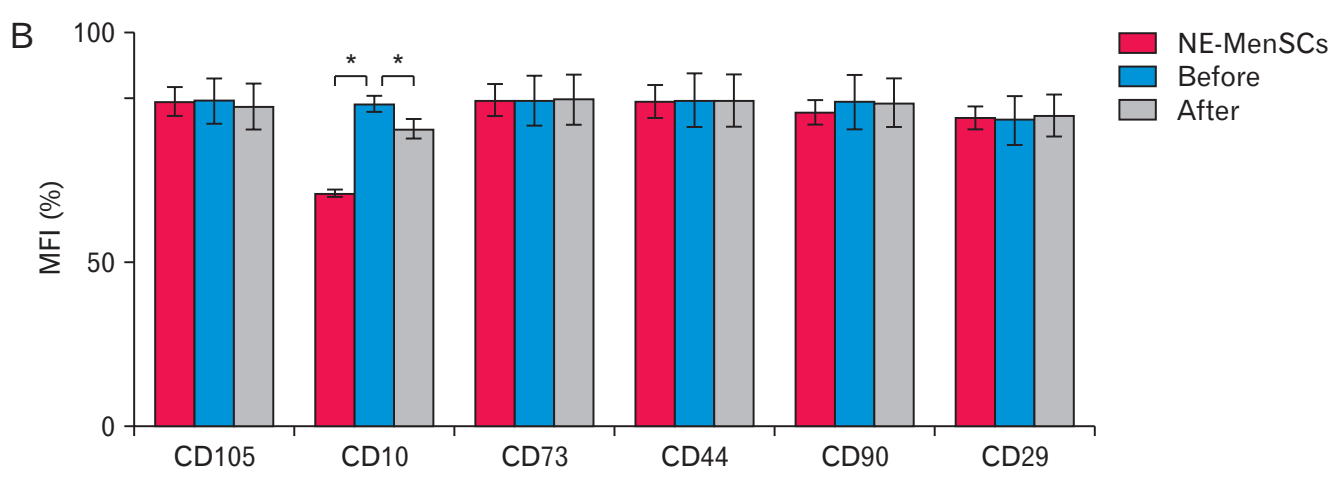

Fig. 3. (A) The expression of menstrual blood-derived mesenchymal stem cells (MenSCs) surface markers was detected by flow cytometry so that they were positive for CD90, CD10, CD 44, CD29, CD73, and CD105, while negative for CD34, CD133, CD45, and CD38. (B) MFI was used to compare E-MenSCs with NE-MenSCs and E-MenSCs after conditioned medium treatment. MFI, mean fluorescence intensity; E-MenSCs, endometriosis derived MenSCs; NE-MenSCs, non endometriosis-derived MenSCs. 
changed under treatment of CM derived from NE-MenSCs into a more elongated, spindle-shaped morphology (Fig. 2C).

\section{Expression of specific mesenchymal stem cell markers}

In order to characterize MenSCs, flow cytometry assay was performed in passage 3 for E-MenSCs (before and after treatment) and NE-MenSCs, and our results showed that both MenSC types (E-MenSC, NE-MenSCs) were positive for CD29, CD90, CD117, CD105, CD44, CD73, and CD10, and negative for CD34, CD45, CD133, CD38 (Fig. 3A).

To compare E-MenSCs and NE-MenSCs concerning the expression level of $\mathrm{CD}$ markers, we used the mean fluorescence intensity (MFI). In our results, CD10 marker expression was significantly higher for E-MenSCs compared with that of NE-MenSCs. Also, after CM treatment, there was significantly decrease in CD10 expression for E-MenSCs $(P<0.05$; Fig. 3B).

\section{Pro-inflammatory genes expression under MenSCs- CM treatment}

In this study E-MenSCs were treated with conditioned medium derived from NE-MenSCs of 3rd passage, for 2, 4, and 6 days. According to the gene expression analysis, the considerable effect was related to 4 days of CM treatment and data from 2 and 6 days of treatment were omitted and not shown. Quantitative RT-PCR analysis of IL-1 $\beta$, COX-2, HIF, TNF- $\alpha$ and NF- $\kappa$ B genes was performed in E-MenSCs in before and after CM treatment, as well as in NE-MenSCs as

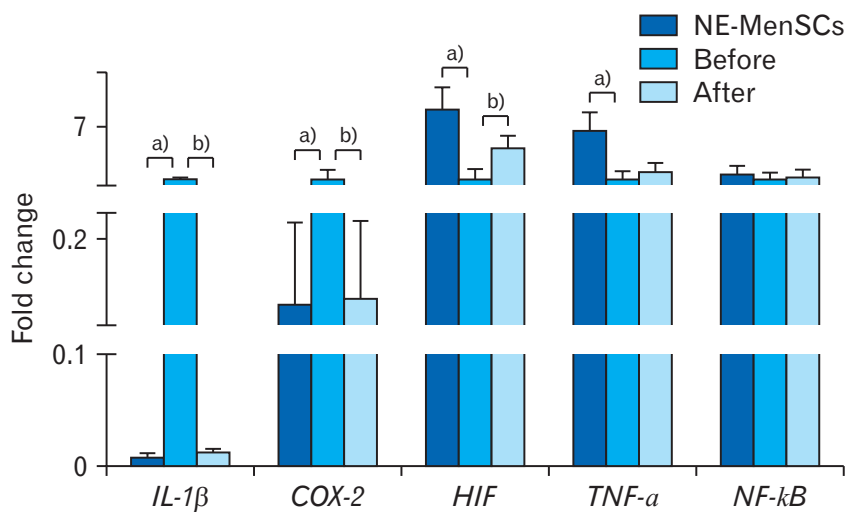

Fig. 4. Comparison of the inflammatory genes expression in NEMenSCs, E-MenSCs (before), and E-MenSCs after conditioned medium (CM) treatment (after) by real-time PCR analysis. ${ }^{\text {a) }}$ Significant difference between NE-MenSCs versus E-MenSCs. ${ }^{\text {b) }}$ Significant difference between before versus after CM treatment. NE-MenSCs, non-endometriosis derived menstrual blood-derived mesenchymal stem cells; E-MenSCs, endometriosis derived menstrual blood-derived mesenchymal stem cells. control group. As shown in Fig. 4, the expression of IL-1 $\beta$ in E-MenSCs was significantly higher compared to NE-MenSCs $(1.00 \pm 0.1$ vs. $0.008 \pm 0.003 ; P<0.001)$, that after $C M$ treatment it was surprisingly decreased $(0.013$ vs. $1.00 ; P<0.001)$ so that was similar to NE-MenSCs $(0.013 \pm 0.002$ vs. $0.008 \pm 0.003$; $P>0.627)$. Similarly, the expression of COX2 in E-MenSCs was significantly higher compared to NE-MenSCs $(1.00 \pm 1.04$ vs. $0.125 \pm 0.09 ; P<0.001)$, that was significantly decreased after $\mathrm{CM}$ treatment $(0.132 \pm 1.08 ; P<0.001)$, which was surprisingly similar to NE-MenSCs $(0.013 \pm 0.002$ vs. $0.008 \pm 0.003$; $P<0.001)$. HIF- $1 \alpha$ gene expression was significantly lower in E-MenSCs compared to NE-MenSCs $(1.00 \pm 1.8$ vs.8.9 \pm 2.4 ; $P<0.001)$, whereas, in treated E-MenSCs, it was significantly up-regulated $(1.00 \pm 1.04$ vs.4.55 $\pm 1.36 ; P<0.001)$. In the case of TNF- $\alpha$ gene, lower expression was observed in E-MenSCs in comparison with NE-MenSCs $(1.00 \pm 1.04$ vs. $6.54 \pm 1.96$; $P<0.001)$. TNF- $\alpha$ expression was increased after CM treatment but this change was not significant. There was no significant difference in $N F-\kappa B$ gene expression between groups.

\section{MenSCs-CM regulates stemness genes expression}

As shown in Fig. 5, the expression of some stemness genes was compared between E-MenSCs (before and after CM treatment) and NE-MenSCs. OCT4 expression showed a significant decrease in E-MenSCs compared to NE-MenSCs $(1.00 \pm 1.04$ vs. $42.22 \pm 7.4 ; P<0.001)$, while under $\mathrm{CM}$ treatment it was significantly up-regulated similar to NE-Men-

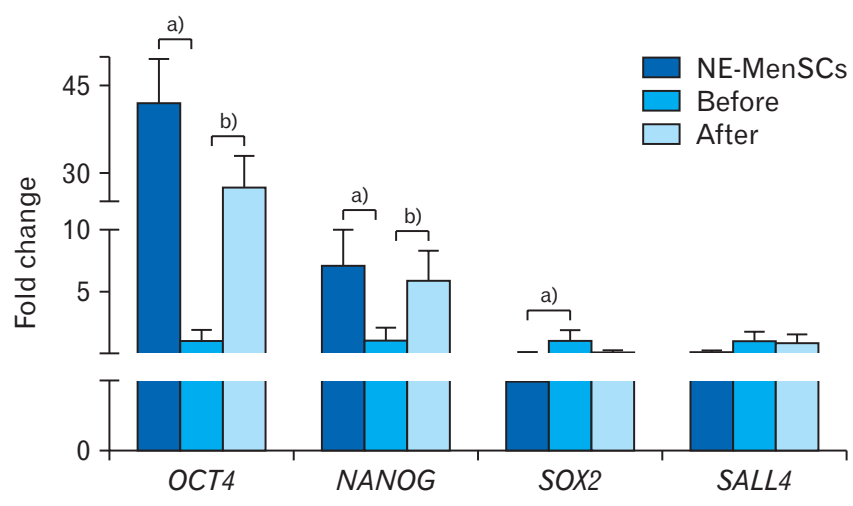

Fig. 5. Comparison of stemness genes expression in NE-MenSCs, E-MenSCs (before), and E-MenSCs after conditioned medium (CM) treatment (after) by real-time PCR analysis. ${ }^{\text {a) }}$ Significant difference between NE-MenSCs versus E-MenSCs. ${ }^{\text {b) }}$ Significant difference between before versus after CM treatment. NE-MenSCs, nonendometriosis derived menstrual blood-derived mesenchymal stem cells; E-MenSCs, endometriosis derived menstrual blood-derived mesenchymal stem cells. 
SCs. $(1.00 \pm 1.04$ vs. $27.63 \pm 5.32 ; P<0.001)$. Similar to OCT4, in the E-MenSCs, NANOG expression was significantly lower than NE-MenSCs $(1.00 \pm 1.04$ vs. $7.1 \pm 2.8 ; P<0.001)$. NANOG was also significantly increased in E-MenSCs after CM treatment and became almost similar to NE-MenSCs $(1.00 \pm 1.04$, 5.99 $\pm 2.3,7.1 \pm 2.8 ; P<0.001)$. In E-MenSCs, SOX2 expression was significantly higher than NE-MenSCs $(1.00 \pm 0.88$ vs. $0.05 \pm 0.04 ; P<0.05)$. Sox 2 was significantly down-regulated in E-MenSCs after CM treatment $(0.14 \pm 0.08 ; P<0.001)$, similar to NE-MenSCs with no significant difference between treated E-MenSCs and NE-MenSCs $(P>0.235)$. Furthermore, SALL4 expression show higher expression in E-MenSCs compared to NE-MenSCs $(1.00 \pm 0.74$ vs. $0.13 \pm 0.09 ; P<0.001)$. No significant difference was observed between E-MenSCs and treated E-MenSCs, so, SALL4 had no difference under CM treatment.

\section{Discussion}

In the present research study, we evaluated the morphological alterations, and gene expression analysis in E-MenSCs-CM treated endometriosis cells compared with untreated controls. We observed that NE-MenSCs-CM could have a considerable effect on the phenotype and expression level of inflammatory and stemness genes of menstrual bloodderived stem cells of endometriosis patients (E-MenSCs).

Recent studies reported many morphological alterations in various cancer cells treated with condition medium of mesenchymal stem cells $[23,24]$. Similar to the study of Nikoo et al. [25], we also observed that NE-MenSCs illustrated a more elongated, spindle-shaped morphology similar to fibroblast-like cells, whereas E-MenSCs did not, and surprisingly the morphology of E-MenSCs changed in the presence of CM derived from NE-MenSCs into a more elongated, spindle-shaped morphology.

In the previous studies, it was demonstrated that some pro-inflammatory genes including IL- 1 and COX- 2 can demonstrate a significantly dysregulated expression in some of tissues of endometriosis patients $[10,12,26-$ 28]. For instance, Chalpe et al. (2015) [26] demonstrated that pro-inflammatory genes, including IL-1 $\beta$ induce differential expressions in endometrial stromal cells. Our results indicated that MenSCs-CM significantly inhibited the expression of IL- $1 \beta$ and COX-2 in endometriosis cells. This result indicated that MenSCs-CM may inhibit the inflammation of endometriosis cells by inhibition of COX-2 expression.

On the other hand, we did not observe a significant difference in the NF- $\kappa \mathrm{B}$ gene expression in E-MenSCs compared to NE-MenSCs. Consistent with previous results, it is possible that NF- $\mathrm{KB}$ activates epithelial cells proliferation and migration most likely via Notch signaling activation resulting in enhancement of re-epithelialization [29]. Given that there is no change in epithelial cell proliferation and epithelialization in endometriosis, it seems that NF- $\kappa \mathrm{B}$ could not have significantly different expression in endometriosis.

Besides, regarding the generally accepted theories reported for the causes of endometriosis include retrograde menstruation, the importance of mesenchymal stem cells present in retrograde menstrual blood and their stemness gene expression were analyzed [30]. Also, previous studies demonstrated that OCT4 and NANOG played an important role in cell proliferation and invasion of various cancer cells [14]. Moreover, the OCT4 and NANOG genes are overexpressed in approximately all human cancers and considered as potential targets for cancer therapy. In contrast to cancer cell studies, we observed lower expression level of NANOG and OCT4 in E-MenSCs in comparison with NE-MenSCs, that treatment of the E-MenSCs with MenSCs-CM resulted in their increased expression. Some Studies have shown SALL4 upregulatetion in endometriosis carcinoma and endometrial cancer cancer [14, 31], but we did not see a significant increase in endometriosis patients.

Moreover, it was cleared that COX-2 upregulation could be observed in endometriotic stromal cells due to the suppression of OCT4 in these stem cells by pro-inflammatory cytokines [32]. Considering in vitro findings, it was identified that COX-2 expression could be increased in the eutopic and ectopic endometrium of endometriosis patients $[10,12$, $27,28]$. These studies were consistent with our study results.

In previous studies, it was observed that some stemness genes, including SOX2 and NANOG demonstrate a significantly higher expression in some tissues of endometriosis patients $[7,14]$. For example, a study revealed that the SOX2 stemness gene has an aberrant expression in endometriosis [33]. Furthermore, Song et al. (2014) [14] reported that SOX2 and NANOG genes were overexpressed in ovarian endometriosis. In line with other studies, we observed that SOX2 had a high expression level in E-MenSCs, which was decreased after treatment with the CM derived from NE-MenSCs.

Given that OCT4 stemness gene has aberrant expression in endometriotic tissues, and considering the influence of 
HIF-2 $\alpha$ transcription factor on the expression of OCT4 [34], Our results also showed a coordinated relationship between the expression of these genes.

On the other hand, in a research study, the MFI was used to evaluate the expression of CD10. The results obtained from this study indicated that CD10 expression was considerably higher in stromal stem cells from endometriosis compared with that of non-endometriosis [25]. Our findings are in agreement with the results obtained from this study.

On the whole, the present study outcomes recommend that CM derived from NE-MenSCs could be exerted as an effective option to influence the expression of genes related to endometriosis formation, such as inflammatory and stemness genes. Nevertheless, further experiments are required in order to profit from these observations. Understanding and identification of the most important contents (extracellular vesicles, microRNAs, etc.) of the NEMenSCs-derived CM is an essential question that should be answered in the future research studies.

\section{ORCID}

Seyedeh Saeideh Sahraei:

https://orcid.org/0000-0002-4943-5748

Ali Kowsari: https://orcid.org/0000-0002-0397-8982

Faezeh Davoodi Asl:

https://orcid.org/0000-0001-7212-0843

Mohsen Sheykhhasan:

https://orcid.org/0000-0002-2522-4292

Leila Naserpoor: https://orcid.org/0000-0003-0565-1729

Azar Sheikholeslami:

https://orcid.org/0000-0001-8277-6539

\section{Author Contributions}

Conceptualization: AS, SSS. Data acquisition: SSS, AK, FDA. Data analysis or interpretation: AS, SSS, AK. Drafting of the manuscript: SSS, MS, AS, LN. Critical revision of the manuscript: MS, AS. Approval of the final version of the manuscript: all authors.

\section{Conflicts of Interest}

No potential conflict of interest relevant to this article was reported.

\section{Acknowledgements}

This work was supported by grants from the Academic Center for Education, Culture and Research, Qom Branch, Qom, Iran. In addition, we are grateful to all the women who participated in this study. We also thank the staff at the midwifery section of infertility treatment clinic of ACECR.

\section{References}

1. Zondervan KT, Becker CM, Koga K, Missmer SA, Taylor RN, Viganò P. Endometriosis. Nat Rev Dis Primers 2018;4:9.

2. Chen L, Qu J, Xiang C. The multi-functional roles of menstrual blood-derived stem cells in regenerative medicine. Stem Cell Res Ther 2019;10:1.

3. Bellelis P, Podgaec S, Abrão MS. Environmental factors and endometriosis. Rev Assoc Med Bras (1992) 2011;57:448-52.

4. Persoons E, De Clercq K, Van den Eynde C, Pinto SJPC, Luyten K, Van Bree R, Tomassetti C, Voets T, Vriens J. Mimicking Sampson's retrograde menstrual theory in rats: a new rat model for ongoing endometriosis-associated pain. Int J Mol Sci 2020;21:2326.

5. Lv H, Hu Y, Cui Z, Jia H. Human menstrual blood: a renewable and sustainable source of stem cells for regenerative medicine. Stem Cell Res Ther 2018;9:325.

6. Bozorgmehr M, Gurung S, Darzi S, Nikoo S, Kazemnejad S, Zarnani AH, Gargett CE. Endometrial and menstrual blood mesenchymal stem/stromal cells: biological properties and clinical application. Front Cell Dev Biol 2020;8:497.

7. Chang JH, Au HK, Lee WC, Chi CC, Ling TY, Wang LM, Kao SH, Huang YH, Tzeng CR. Expression of the pluripotent transcription factor OCT4 promotes cell migration in endometriosis. Fertil Steril 2013;99:1332-9.e5.

8. Deldar Y, Zarghami F, Pilehvar-Soltanahmadi Y, Dadashpour M, Zarghami N. Antioxidant effects of chrysin-loaded electrospun nanofibrous mats on proliferation and stemness preservation of human adipose-derived stem cells. Cell Tissue Bank 2017;18:475-87.

9. Fakih H, Baggett B, Holtz G, Tsang KY, Lee JC, Williamson HO. Interleukin-1: a possible role in the infertility associated with endometriosis. Fertil Steril 1987;47:213-7.

10. Banu SK, Lee J, Speights VO Jr, Starzinski-Powitz A, Arosh JA. Cyclooxygenase- 2 regulates survival, migration, and invasion of human endometriotic cells through multiple mechanisms. Endocrinology 2008;149:1180-9.

11. Kyama CM, Overbergh L, Debrock S, Valckx D, Vander Perre S, Meuleman C, Mihalyi A, Mwenda JM, Mathieu C, D’Hooghe TM. Increased peritoneal and endometrial gene expression of biologically relevant cytokines and growth factors during the menstrual phase in women with endometriosis. Fertil Steril 2006;85:1667-75. 
12. Wu MH, Hsiao KY, Tsai SJ. Endometriosis and possible inflammation markers. Gynecol Minim Invasive Ther 2015;4:617.

13. Proestling K, Birner P, Balendran S, Nirtl N, Marton E, Yerlikaya G, Kuessel L, Reischer T, Wenzl R, Streubel B, Husslein $\mathrm{H}$. Enhanced expression of the stemness-related factors OCT4, SOX15 and TWIST1 in ectopic endometrium of endometriosis patients. Reprod Biol Endocrinol 2016;14:81.

14. Song Y, Xiao L, Fu J, Huang W, Wang Q, Zhang X, Yang S. Increased expression of the pluripotency markers sex-determining region Y-box 2 and Nanog homeobox in ovarian endometriosis. Reprod Biol Endocrinol 2014;12:42.

15. Park JH, Daheron L, Kantarci S, Lee BS, Teixeira JM. Human endometrial cells express elevated levels of pluripotent factors and are more amenable to reprogramming into induced pluripotent stem cells. Endocrinology 2011;152:1080-9.

16. Siu MK, Wong ES, Kong DS, Chan HY, Jiang L, Wong OG, Lam EW, Chan KK, Ngan HY, Le XF, Cheung AN. Stem cell transcription factor NANOG controls cell migration and invasion via dysregulation of E-cadherin and FoxJ1 and contributes to adverse clinical outcome in ovarian cancers. Oncogene 2013;32:3500-9.

17. Zhang W, Sui Y, Ni J, Yang T. Insights into the Nanog gene: a propeller for stemness in primitive stem cells. Int J Biol Sci 2016;12:1372-81.

18. Forghanifard MM, Moghbeli M, Raeisossadati R, Tavassoli A, Mallak AJ, Boroumand-Noughabi S, Abbaszadegan MR. Role of SALL4 in the progression and metastasis of colorectal cancer. J Biomed Sci 2013;20:6.

19. Ardalan Khales S, Abbaszadegan MR, Abdollahi A, Raeisossadati R, Tousi MF, Forghanifard MM. SALL4 as a new biomarker for early colorectal cancers. J Cancer Res Clin Oncol 2015;141:229-35.

20. Joseph A, Baiju I, Bhat IA, Pandey S, Bharti M, Verma M, Pratap Singh A, Ansari MM, Chandra V, Saikumar G, Amarpal, Taru Sharma G. Mesenchymal stem cell-conditioned media: a novel alternative of stem cell therapy for quality wound healing. J Cell Physiol 2020;235:5555-69.

21. Noverina R, Widowati W, Ayuningtyas W, Kurniawan D, Afifah E, Laksmitawati DR, Rinendyaputri R, Rilianawati R, Faried A, Bachtiar I, Wirakusumah FF. Growth factors profile in conditioned medium human adipose tissue-derived mesenchymal stem cells (CM-hATMSCs). Clin Nutr Exp 2019;24:3444.

22. Sefati N, Norouzian M, Abbaszadeh HA, Abdollahifar MA, Amini A, Bagheri M, Aryan A, Fadaei Fathabady F. Effects of bone marrow mesenchymal stem cells-conditioned medium on tibial partial osteotomy model of fracture healing in hypothyroidism rats. Iran Biomed J 2018;22:90-8.

23. Gauthaman K, Fong CY, Arularasu S, Subramanian A, Biswas A, Choolani M, Bongso A. Human Wharton's jelly stem cell conditioned medium and cell-free lysate inhibit human osteosarcoma and mammary carcinoma cell growth in vitro and in xenograft mice. J Cell Biochem 2013;114:366-77.

24. Gauthaman K, Fong CY, Suganya CA, Subramanian A, Biswas A, Choolani M, Bongso A. Extra-embryonic human Wharton's jelly stem cells do not induce tumorigenesis, unlike human embryonic stem cells. Reprod Biomed Online 2012;24:235-46.

25. Nikoo S, Ebtekar M, Jeddi-Tehrani M, Shervin A, Bozorgmehr M, Vafaei S, Kazemnejad S, Zarnani AH. Menstrual bloodderived stromal stem cells from women with and without endometriosis reveal different phenotypic and functional characteristics. Mol Hum Reprod 2014;20:905-18.

26. Chalpe A, Law C, Dumdie J, Hansen K, Eyster K. TNF $\alpha$ and IL1 $\beta$ stimulate differential gene expression in endometrial stromal cells. Adv Biol Chem 2015;5:126-41.

27. Ota H, Igarashi S, Sasaki M, Tanaka T. Distribution of cyclooxygenase-2 in eutopic and ectopic endometrium in endometriosis and adenomyosis. Hum Reprod 2001;16:561-6.

28. Matsuzaki S, Canis M, Pouly JL, Wattiez A, Okamura K, Mage G. Cyclooxygenase-2 expression in deep endometriosis and matched eutopic endometrium. Fertil Steril 2004;82:1309-15.

29. Nickoloff BJ, Qin JZ, Chaturvedi V, Denning MF, Bonish B, Miele L. Jagged-1 mediated activation of notch signaling induces complete maturation of human keratinocytes through NFkappaB and PPARgamma. Cell Death Differ 2002;9:842-55.

30. Santamaria X, Massasa EE, Taylor HS. Migration of cells from experimental endometriosis to the uterine endometrium. Endocrinology 2012;153:5566-74.

31. Liu L, Zhang J, Yang X, Fang C, Xu H, Xi X. SALL4 as an epithelial-mesenchymal transition and drug resistance inducer through the regulation of c-Myc in endometrial cancer. PLoS One 2015;10:e0138515.

32. Lin SC, Li YH, Wu MH, Chang YF, Lee DK, Tsai SY, Tsai MJ, Tsai SJ. Suppression of COUP-TFII by proinflammatory cytokines contributes to the pathogenesis of endometriosis. J Clin Endocrinol Metab 2014;99:E427-37.

33. Götte M, Wolf M, Staebler A, Buchweitz O, Kiesel L, Schüring AN. Aberrant expression of the pluripotency marker SOX-2 in endometriosis. Fertil Steril 2011;95:338-41.

34. Zhu C, Yu J, Pan Q, Yang J, Hao G, Wang Y, Li L, Cao H. Hypoxia-inducible factor-2 alpha promotes the proliferation of human placenta-derived mesenchymal stem cells through the MAPK/ERK signaling pathway. Sci Rep 2016;6:35489. 